\title{
The Use of New Approaches to Estimating the Coherence Properties of Mutually Orthogonal Beams
}

\author{
O.V. Angelsky*, C.Yu. Zenkova, M.P.Gorsky, I.V. Soltys and P.O. Angelsky
}

Chernivtsy National University, Kotsyubinsky, 2, Chernivtsy, Ukraine

\begin{abstract}
A theoretical model for calculating the motion dynamics of the particles of different light-scattering mechanisms in the energy inhomogeneous optical field is suggested. The direct relationship between the motion velocity of the tested particles in the created field and the degree of coherence of mutually orthogonal linearly polarized plane waves is demonstrated. The possibilities of using the normalized value of the averaged velocity for particles of the $\lambda / 100$ sizes to define the degree of coherence of mutually orthogonal linearly polarized fields are shown.
\end{abstract}

Keywords: Degree of coherence, nanoparticles, Poynting vector, light scattering, polarization, optical force, energy density.

\section{INTRODUCTION}

Taking into consideration the modern tendencies for developing nanotechnologies, much attention is paid to studying the behaviour of micro- and nanoparticles and the ways of controlling them [1]. To do this the micromanipulators and micromachines are needed. The trapping and transportation $[2,3]$ of particles, molecules, atoms, biological objects, such as DNA molecules, organelle cells, increase the potentiality of biomedicine in diagnosing the pathology of human organs [4-10]. As a rule, beams of various nature, such as Gaussian beams [11, 12], bottle beams [13], zero-order Bessel beams [14], self-focused laser beams [15] and even evanescent fields $[16,17]$, reflecting different forms of energy circulation $[18,19]$, are used for these purposes.

The formation of energy redistribution and energy circulation fields can occur in a simpler case as well. This research proposes the interaction scheme of two mutually orthogonal linearly polarized waves in the incidence plane, where the modulation of polarization and the modulation of the energy volume density occur simultaneously in the observation plane $[19,20]$.

It is difficult to estimate the degree of coherence in such a modulated situation, because it is impossible to use classical estimation methods in this case. We introduced a new parameter, the visibility modulation depth (VMD), in our papers $[21,22]$. This parameter enabled to approach to the solution of the set task. However, the problems associated with registering the polarization information on polarization sensitive materials and the search for corresponding materials cause the need for new ways and methods of estimating the degree of coherence. The research presupposes the use of the ability of inhomogeneous optical fields to control the tested particles in the modulated situation. The investigation

*Address correspondence to this author at the Chernivtsy National University, Kotsyubinsky, 2, Chernivtsy, Ukraine; Tel: +38(03722)44730;

Fax:+38(03722)44730; E-mail: angelsky@itf.cv.ua is carried out with the aim to define the coherence properties of those fields that form the energy inhomogeneous optical field.

In this case the depth of the energy volume density modulation essentially depends on the degree of coherence of interacting waves. The way of monitoring particles of the Rayleigh light scattering mechanism in the energy inhomogeneous optical field is proposed for investigation. In this case the complex optical force affecting these particles is taken into consideration. The task of defining the degree of coherence of mutually orthogonal linearly-polarized plane waves using the particle motion velocity in the created optical field is solved as well.

\section{THE STATEMENT OF THE PROBLEM}

At present optical traps and micromanipulators are created taking into consideration the basic properties of laser radiation. In this case the components of the optical force compete with each other, i.e. the components of radiation pressure, scattering force and gradient force [23]. The scattering force is proportional to the radiation intensity and acts in the direction of the incident laser beam. The gradient component acts in the direction of the gradient intensity. These components may create the stable point, which is localized near the focus. If one of the components is larger or smaller than the other one, the equilibrium point is missing and a directional particle motion is observed. Thus, the availability of field pressure, which is connected with the existence of the internal angular momentum of the field, causes the arising of the energy flux, because the momentum density and the density of the energy flux are proportional to each other [24]. The momentum of the field passed on to a particle causes the movement of that particle.

The calculation of the optical force, affecting particles of different light scattering mechanisms, depends on the sizes and properties of the tested particles [25], as well as on the properties and characteristics of the optical field where the particles are localized. 
We consider the particles, for which the Rayleigh lightscattering mechanism can be applied. There are particles, whose size (radius $r$ ) is much smaller than that of the light wavelength, or, according to the more accurate definition $r<<\lambda / 20$. In literature these particles are named Rayleigh particles [26]. In this research we assume that the size of the particles amounts to $\lambda / 100$. The interaction of these particles with the electromagnetic wave is described within the framework of the Rayleigh light scattering mechanism. In this case the approximation of the point dipole is introduced. The other group of particles are those whose size (radius) is defined as $\lambda / 5$. The physical mechanism, setting the interaction of particles with the optical field, is explained in the context of the Rayleigh light scattering approximation, and corresponds to the Mie theory.

The consideration of each component of the optical force and the calculation of the gradient, scattering and absorbing components are important for the given types of particles.

In this research the basic elements of the approach suggested in [27] are used, with the interferential scheme of interaction of two plane waves of equal amplitudes linearly polarized at the incidence plane, with an interference angle of $90^{\circ}$ taken into account (Fig. 1).

When accounting the superposition of two mutually orthogonal linearly polarized fields, the modulation of the averaged Poynting vector value, i.e. the spatial modulation of energy density occurs in the observation plane. The optical force, expressed by the Poynting vector, forms the particle distribution in this plane. Moreover, the change of the degree of coherence leads to the change of the modulation depth of the energy volume density, which, in its turn, determines the optical force value and the peculiarities of particle motion.

Generally, the forces exerted on the particles by a laser beam depend on the properties of both the beam and the particles.

We consider the behavior of the tested particles located in the inhomogeneous optical field at room temperature. According to the experiment proposed in [19], these are the particles of golden hydrosol of about $1 \mathrm{~nm}$ and $100 \mathrm{~nm}$ in size. The mechanical effect of spin flows [28, 29] on the motion of particles is not analyzed in this paper.

Let us discuss the formation of the optical force $\mathbf{F}_{\text {opt }}$ affecting the tested particles. The direction of the optical force gradient component is stipulated by the distribution gradient of the energy volume density. The direction of the scattering and absorbing components sets the direction of the energy flow propagation. At the same time the direction of the reflecting component is set by the orientation of the particle surface.

The gradient component of the optical force, taking into account the polarization of the molecule $\alpha=\alpha^{\prime}+i \alpha^{\prime \prime}$, can be calculated as $\mathbf{F}_{\text {grad }}=-\frac{1}{2} n \alpha \nabla E^{2}$. The polarization of the molecule $\alpha$ can be derived from the Clausius-Mossoti equation as $\alpha=3 V \frac{\varepsilon_{p}-\varepsilon_{m}}{\varepsilon_{p}+2 \varepsilon_{m}}$, where $\varepsilon_{p}\left(\varepsilon_{m}\right)$ are the values of the dielectric permittivity of a gold particle (a surrounding me- dium) correspondingly. Taking into consideration that for golden particles in water $n=0.32+2.65 i$ [30], the real and imaginary parts of the dielectric permittivity are calculated using the Kramers-Kronig relation. As it was obtained in [31] $\mathbf{F}_{\text {grad }}=-\frac{1}{2} n \alpha^{\prime} \nabla E^{2}=-\frac{2 \pi n \alpha^{\prime}}{c} \sqrt{\frac{\mu}{\varepsilon}} \nabla S$.

Thus, one can find the gradient of the field for the general case. The gradient of an arbitrary function can be calculated at any point of the plane, including the observation plane. It is known that the modulus of an arbitrary function gradient is equal to the greatest change of the function in the chosen point.

Thus, it is possible to calculate the gradient distribution of the volume energy density.

$\nabla S=\vec{i} \frac{\partial S}{\partial x}+\vec{k} \frac{\partial S}{\partial z}$, assuming that the distribution gradient of the volume energy density takes place in the plane $x, z$.

Here

$S=\frac{\sqrt{\varepsilon \varepsilon_{0}}}{\sqrt{\mu \mu_{0}}} \sum_{i, j}\left\{\begin{array}{l}\varphi_{i j}^{(1)}(\mathbf{r})+\varphi_{i j}^{(2)}(\mathbf{r})+2 \sqrt{\operatorname{tr}\left[W\left(\mathbf{r}_{1}, \mathbf{r}_{1}, 0\right)\right] \mathrm{tr}\left[W\left(\mathbf{r}_{2}, \mathbf{r}_{2}, 0\right)\right]} \cdot \\ \left|\eta_{i j}^{(1,2)}\right| \cos \left[\alpha_{i j}^{(1,2)}\right] \cdot \cos \left[\delta_{e}\right]\end{array}\right\}$ is a time-

averaged density of the energy current $[32,33]$. The mutual coherence matrix $W\left(\mathbf{r}_{1}, \mathbf{r}_{2}, \tau\right)$ describes the coherence properties of the vector optical fields and characterizes the correlation of the fields at two different spatial points $\mathbf{r}_{1}$ and $\mathbf{r}_{2}[33$, 34]. Here the components of the degree of mutual coherence of the field are determined $[33,34]$ as

$$
\eta_{i j}\left(\mathbf{r}_{1}, \mathbf{r}_{2}, \tau\right)=\frac{\left.<E_{i}^{(1)} \mathbf{r}_{1}, \tau\right) E_{j}^{(2)^{*}}\left(\mathbf{r}_{2}, \tau\right)>}{\sqrt{\left.\sum_{i j}<E_{i}^{(1)} \mathbf{r}_{1}, 0\right) E_{i}^{(2)^{*}}\left(\mathbf{r}_{1}, 0\right)><E_{j}^{(1)}\left(\mathbf{r}_{2}, 0\right) E_{j}^{(2)^{*}}\left(\mathbf{r}_{2}, 0\right)>}}
$$

We assume that $\tau=0$. This enables us to estimate the spatial coherence of the optical field.

$\alpha_{i j}^{(1,2)}-$ is the argument of $\eta_{i j}^{(1,2)}$ which determines the phase difference between $i$ - and $j$ - components of the field, where $i, j=x, z ; \delta_{e}-$ is the phase difference between the beams (in this case - their electrical parts) at the registration plane.

Let us assume that the coordinates of the arbitrary point in the region of minimum values of volume energy density are $x_{\text {min, }} z_{\text {min }}$. In order to define the value of the gradient $\nabla S$ at the arbitrary point $m$, with coordinates $x_{m}, z_{m}$ in the observation plane, the remoteness of the given point relative to the nearest point is marked off in the minimum energy region.

Then for the $m$ - point we can write down the following:

$$
|\nabla S|_{m}=\sqrt{\left(\frac{\partial S_{m}}{\partial x_{m}}\right)^{2}+\left(\frac{\partial S_{m}}{\partial z_{m}}\right)^{2}}=\sqrt{\left(\frac{\Delta S_{m}}{\Delta x_{m}}\right)^{2}+\left(\frac{\Delta S_{m}}{\Delta z_{m}}\right)^{2}}=\Delta S_{m} \sqrt{\left(\frac{1}{\Delta x_{m}}\right)^{2}+\left(\frac{1}{\Delta z_{m}}\right)^{2}},
$$

where $\Delta x_{m}=x_{\min }-x_{m}, \Delta z_{m}=z_{\min }-z_{m}, \Delta S_{m}=S_{\min }-S_{m}$.

$$
S_{\min }=\frac{\sqrt{\varepsilon \varepsilon_{0}}}{\sqrt{\mu \mu_{0}}} \sum_{i, j}\left\{\varphi_{i j}^{(1)}(\mathbf{r})+\varphi_{i j}^{(2)}(\mathbf{r})-2 \sqrt{\operatorname{tr}\left[W\left(\mathbf{r}_{1}, \mathbf{r}_{1}, 0\right)\right] \operatorname{tr}\left[W\left(\mathbf{r}_{2}, \mathbf{r}_{2}, 0\right)\right]} \cdot \eta_{i j}^{(1,2)}\right\}
$$


$S_{m}=\frac{\sqrt{\varepsilon \varepsilon_{0}}}{\sqrt{\mu \mu_{0}}} \sum_{i, j}\left\{\begin{array}{l}\varphi_{i j}^{(1)}(\mathbf{r})+\varphi_{i j}^{(2)}(\mathbf{r})+2 \sqrt{\operatorname{tr}\left[W\left(\mathbf{r}_{1}, \mathbf{r}_{1}, 0\right)\right] \operatorname{tr}\left[W\left(\mathbf{r}_{2}, \mathbf{r}_{2}, 0\right)\right]} \cdot \\ \eta_{i j}^{(1,2)} \cdot \cos \left[\left(\delta_{e}\right)_{m}\right]\end{array}\right\}$, then

it is possible to calculate

$\Delta S_{m}=-2 \frac{\sqrt{\varepsilon \varepsilon_{0}}}{\sqrt{\mu \mu_{0}}} \sum_{i j} \sqrt{\operatorname{tr}\left[W\left(\mathbf{r}_{1}, \mathbf{r}_{1}, 0\right)\right] \operatorname{tr}\left[W\left(\mathbf{r}_{2}, \mathbf{r}_{2}, 0\right)\right]} \cdot \eta_{i j}^{(1,2)}\left(1-\cos \left[\left(\delta_{e}\right)_{m}\right]\right)$

$=\frac{\sqrt{\varepsilon}}{\sqrt{\mu}} K_{m} \sum_{i j} \eta_{i j}^{(1,2)}$.

Here $K_{m}=-2 \frac{\sqrt{\varepsilon_{0}}}{\sqrt{\mu_{0}}} \sqrt{\operatorname{tr}\left[W\left(\mathbf{r}_{1}, \mathbf{r}_{1}, 0\right)\right] \operatorname{tr}\left[W\left(\mathbf{r}_{2}, \mathbf{r}_{2}, 0\right)\right]} \cdot\left(1-\cos \left[\left(\delta_{e}\right)_{m}\right]\right)$ is the parameter, which characterizers the energy density value for the chosen point $m$ of the optical field, and depends on the phase difference between the initial waves at the corresponding point.

Thus, it can be written as $\Delta S_{m}=\sqrt{\frac{\varepsilon}{\mu}} K_{m} \cdot \eta^{(1,2)}$.

Then the value of the gradient component of the optical force in the scalar form is expressed as $\left(F_{\text {grad }}\right)_{m}=-\frac{2 \pi n \alpha^{\prime}}{c} K_{m} \sqrt{\left(\frac{1}{\Delta x_{m}}\right)^{2}+\left(\frac{1}{\Delta z_{m}}\right)^{2}} \cdot \eta^{(1,2)}$.

The effect of the gradient force is related to the gradient of the energy volume density, and, correspondingly, to the value of the degree of coherence of interacting fields.

According to [26], it is possible to define the scattering and absorbing components of the optical force, using the Rayleigh approximation:

$\mathbf{F}_{\text {scat }}=\frac{n}{c} C_{\text {scat }} \mathbf{S}$, where $C_{\text {scat }}=\frac{k^{4}}{6 \pi \varepsilon_{0}^{2}}|\alpha|^{2}, k=2 \pi / \lambda$

$\mathbf{F}_{a b s}=\frac{n}{c} C_{a b s} \mathbf{S}$, where $C_{a b s}=\frac{k}{\varepsilon_{0}}\left|\alpha^{\prime \prime}\right|^{2}$

Considering all the above described components of the optical force [35] can be rewritten as follows:

$\mathbf{F}_{\text {opt }}=\mathbf{F}_{\text {grad }}+\mathbf{F}_{\text {scat }}+\mathbf{F}_{a b s}$

Depending on the relation between the values of the gradient component of the optical force and the scattering or absorbing components, the particles are either trapped by the field or they move in the direction caused by the direction of the energy flow.

May the analyzed particles be situated in a viscous medium. Let us assume that the mixture consists of a viscous incompressible carrier phase and spherical particles, whose radius is $r$ and whose mass is $M$.

When moving in the liquid, the particle is affected by the friction force, which at the constant value of the velocity $\mathbf{v}$ is defined by the Stokes law as $\mathbf{F}_{s t}=B \mathbf{v}=6 \pi \eta r \mathbf{v}$, where $B$ is the particle friction coefficient, $\eta$ is the dispersive me- dium viscosity, $r$ is the particle radius. Using the basic equation of particles motion in the optical field with consideration of the force of viscous friction [35] can be written as

$M \frac{d \mathbf{v}}{d t}=\mathbf{F}_{o p t}+\mathbf{F}_{s t}$

In the first approximation we ignore the Brownian motion of particles and believe that the particles were motionless before they were moved into the zone of the optical field effect.

We study the particle $m$ with the mass $M$ and the radius $r$, which is situated at the point of the field with coordinates $x_{m}, z_{m}$, and write down the equation of motion for it in the scalar form

$M \frac{d v_{m}}{d t}=-6 \pi \eta r v-\frac{2 \pi n}{c} \alpha^{\prime} K_{m} \eta^{(1,2)} \sqrt{\left(\frac{1}{\Delta x_{m}}\right)^{2}+\left(\frac{1}{\Delta z_{m}}\right)^{2}}+\left\{C_{a b s}+C_{\text {scatt }}\right\} \frac{n}{c} S_{m}$.

When solving the obtained differential equation we get

$$
\begin{aligned}
& v_{m}(t)=\frac{1}{M}\left(e^{-\frac{6 \pi \eta r^{M}}{M} t}-1\right) \times\left\{\frac{2 \pi n}{c} \alpha^{\prime} K_{m} \eta^{(1,2)} \sqrt{\left(\frac{1}{\Delta x_{m}}\right)^{2}+\left(\frac{1}{\Delta z_{m}}\right)^{2}}-\right. \\
& \left\{C_{a b s}+C_{\text {scatt }}\right\} \frac{n}{c} \frac{\sqrt{\varepsilon \varepsilon_{0}}}{\sqrt{\mu \mu_{0}}} \sum_{i, j}\left\{\begin{array}{l}
\varphi_{i j}^{(1)}(\mathbf{r})+\varphi_{i j}^{(2)}(\mathbf{r})+2 \sqrt{\operatorname{tr}\left[W\left(\mathbf{r}_{1}, \mathbf{r}_{1}, 0\right)\right] \operatorname{tr}\left[W\left(\mathbf{r}_{2}, \mathbf{r}_{2}, 0\right)\right]} \\
\eta_{i j}^{(1,2)} \cdot \cos \left[\left(\delta_{e}\right)_{m}\right]
\end{array}\right\} .
\end{aligned}
$$

As it is seen from this expression, the velocity value of each separate particle $v_{m}(t)$ depends on the position of the particle in the optical field.

We perform the averaging over the ensemble of particles localized in the optical field.

Then the motion velocity of $m$ particles averaged over the ensemble of the particles can be written as

$$
\begin{aligned}
& \bar{v}(t)=\frac{1}{M}\left(e^{-\frac{6 \pi \eta r}{M} t}-1\right) \times\left\{\frac{1}{m} \sum_{m} \frac{2 \pi n}{c} \alpha^{\prime} K_{m} \eta^{(1,2)} \sqrt{\left(\frac{1}{\Delta x_{m}}\right)^{2}+\left(\frac{1}{\Delta z_{m}}\right)^{2}}-\right. \\
& \left.\frac{1}{m} \sum_{m}\left[\left\{C_{a b s}+C_{\text {scatt }}\right\} \frac{n}{c} \frac{\sqrt{\varepsilon \varepsilon_{0}}}{\sqrt{\mu \mu_{0}}} \sum_{i, j}\left\{\begin{array}{l}
\varphi_{i j}^{(1)}(\mathbf{r})+\varphi_{i j}^{(2)}(\mathbf{r})+2 \sqrt{\operatorname{tr}\left[W\left(\mathbf{r}_{1}, \mathbf{r}_{1}, 0\right)\right] \operatorname{tr}} \\
{\left[W\left(\mathbf{r}_{2}, \mathbf{r}_{2}, 0\right)\right]} \\
\eta_{i j}^{(1,2)} \cdot \cos \left[\left(\delta_{e}\right)_{m}\right]
\end{array}\right]\right]\right\}
\end{aligned}
$$

From this equation, the influence of the degree of mutual coherence on the value of the averaged velocity of particle motion in the optical field is observed.

To obtain a direct linkage between the averaged velocity of tested particles and the degree of coherence of superposing fields we choose as an example the tested particles characterized by the following set of properties: the Rayleigh mechanism of light scattering is peculiar to them; the values of the scattering and absorbing components of the optical force affecting these particles are much smaller than the value of the gradient component of the optical force.

In this case the value of the averaged motion velocity of nanoparticles in the optical field, or more precisely, the velocity of trapping the given particles by the field is determined by the degree of mutual coherence of superposing fields and is written as 


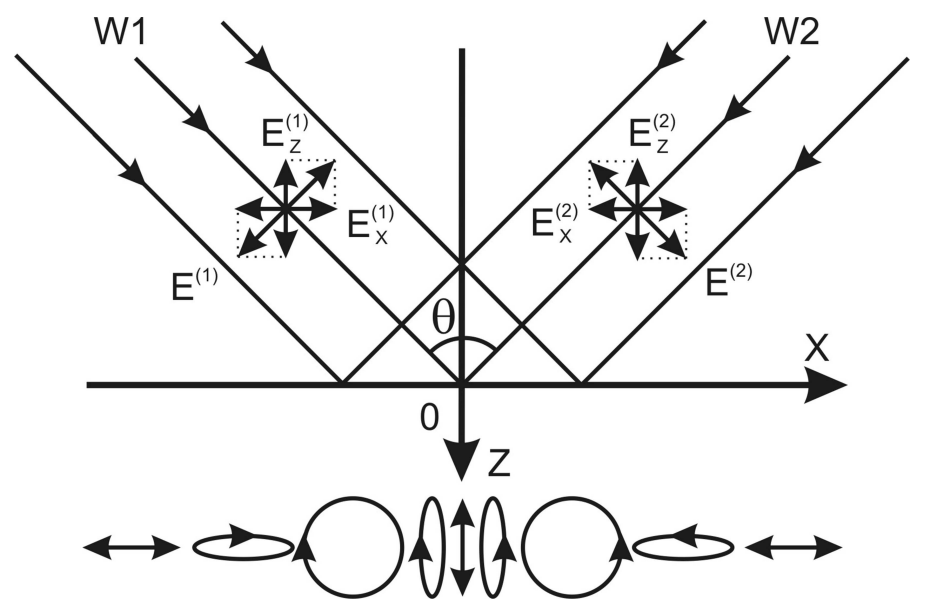

Fig. (1). The superposition of plane waves of equal amplitudes linearly polarized at the incidence plane, with interference angle of $90^{\circ}$. Periodical spatial modulation of polarization $[21,22,30]$ takes place in the plane of incidence.

$\bar{v}(t)=\frac{1}{M}\left(e^{-\frac{6 \pi \eta r}{M} t}-1\right) \frac{2 \pi n}{c} \alpha^{\prime} \eta^{(1,2)} \frac{1}{m} \sum_{m} K_{m} \sqrt{\left(\frac{1}{\Delta x_{m}}\right)^{2}+\left(\frac{1}{\Delta z_{m}}\right)^{2}}$

If we normalize the change of the averaged velocity with time to the maximum of the trapping velocity for each corresponding moment of time obtained in the absolutely coherent field, we get

$\bar{v}_{r e l}(t)=\eta^{(1,2)}$

Thus, the relatively averaged motion velocity of nanometer range particles in the energy inhomogeneous optical field, created by the interaction of partially coherent optical fields converging at the angle of $90^{\circ}$, enables to estimate the degree of mutual coherence of these fields.

\section{THE RESULTS OF SIMULATION}

Using the methods suggested in papers [20, 32, 36], we can demonstrate the motion of particles of various sizes in the optical field, formed by the superposition of coherent and partially coherent beams. To create a more complete picture of particle motion it is necessary to take into consideration the set of forces acting on the side of the liquid, where the particles are maintained in suspension.

The optical field, in which the motion of different kinds of particles can be observed, is created by the interference of two plane waves with different degrees of coherence investigated in the experiment (Fig. 1). Though a homogeneous distribution of intensity is observed in the registration plane of the resulting field, the distribution of the Poynting vector can acquire an inhomogeneous character. It essentially depends upon the degree of coherence of interacting beams.

Let us reproduce the full picture of particle motion in the optical field. The essential polarization of golden particles and the availability of absorption determine the picture of particle motion: (i) the redistribution of particles between the maximum and minimum regions in the energy inhomogeneous optical field, caused by the action of the optical field, and (ii) further behavior of the particles in accordance with their size and the relation between the components of the optical force. The initial conditions determine the distribution of particles by velocity at the beginning of the motion, i.e. at the initial moments of time. Correspondingly, the resulting optical force and the full force, determining the particle motion, depend on localization of the particle. The calculation of the resulting optical force is performed using Exp. (5). In this case in the first approximation the particles are considered motionless at the initial moment.

We shall begin the analysis of particle behavior with simulating the motion of tested particles whose size is about $100 \mathrm{~nm}$.

In the case of incoherent interaction of plane waves in our interference experiment $[34,36]$ there is no spatial modulation of polarization in the observation plane. As it is suggested in the above mentioned papers, the modulation of the Poynting vector is absent as well. The gradient component of the optical force, caused by the gradient of the Poynting vector value (the gradient of the volume energy density), does not change the direction of the particle motion. The particle motion of the offered simulated situation is explained by the presence of the resulting force caused by the scattering and absorbing components of the optical force, which is closely connected with the radiation pressure and the viscous friction on the liquid side. The formation of the resulting optical force affecting the tested particles is presented in Fig. (2). The graphical representations of the optical force (Fig. 2) are obtained by normalizing the force value at a certain moment of time to the force value obtained when absolutely coherent fields interact at the same moment of time.

In the given situation the particles move in the direction of the waves energy propagation in the observation plane. The particle motion velocity depends on the relationship between the field momentum value and viscous friction force.

The account of significant light absorption by golden particles determines the direction of the resulting optical force. The absorption component becomes important for the particles of the chosen size, and with the increase of the size of the particles the importance of the given component increases.

The change of the degree of coherence of the interacting waves leads to the change of the depth of the energy inhomogeneity of the optical field. The modulation of polariza- 


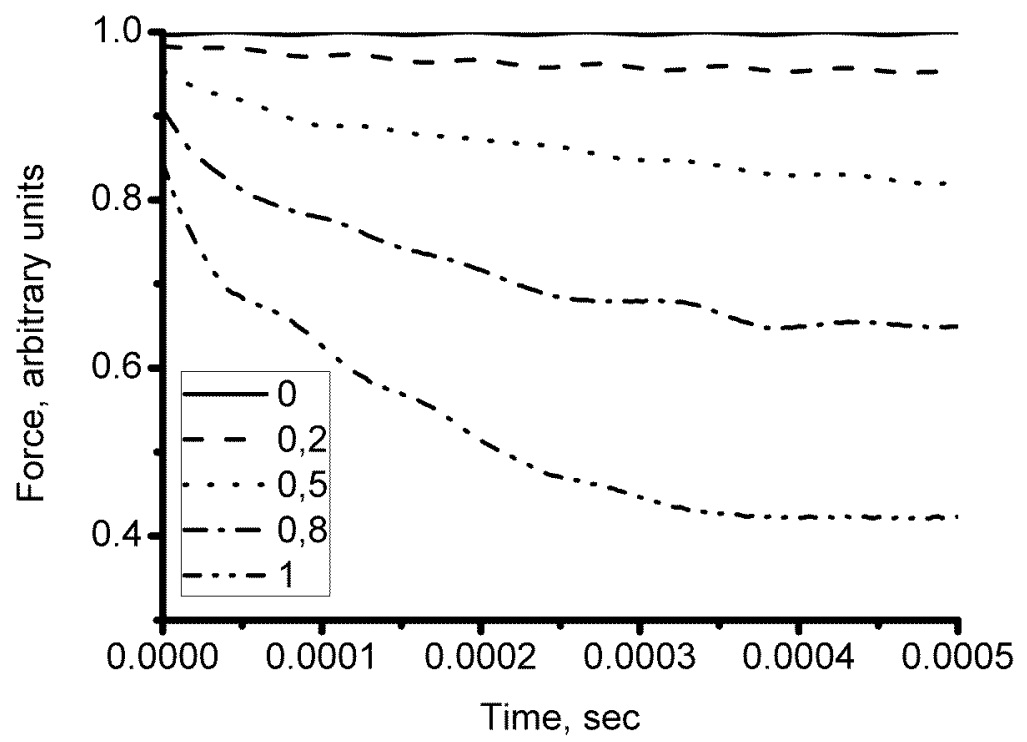

Fig. (2). Changes in the resulting optical force affecting the particles above $\lambda / 5$ in size observed with time and changing the degree of coherence $\left(\eta^{(1,2)}\right)$ of the interacting waves: the legend shows degrees of coherence that correspond to different curves.

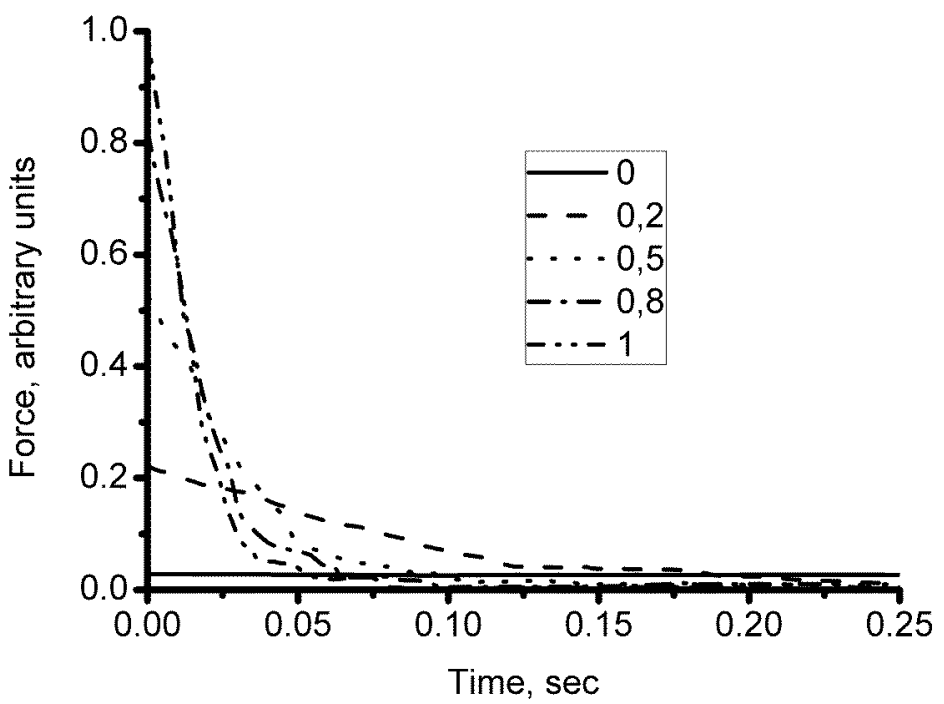

Fig. (3). The change of the optical force affecting Rayleigh particles (the particles above $\lambda / 100$ in size) with time and with the change of the degree of coherence of interacting waves $\left(\eta^{(1,2)}\right)$ : the legend shows degrees of coherence that correspond to different curves.

tion and the modulation of the energy volume density are observed in the observation plane and lead to the arising of the gradient component of the optical force.

As a result, the increase of the degree of coherence produces the decrease of the resulting optical force (Fig. 2) acting on the type of particles under discussion.

Since the gradient force value is rather small in comparison with the other two components of the optical force, the value of this component is not enough to capture the particles. As a result, the particles, according to the main laws of thermodynamics, move to the region of the potential energy minimum. These zones are localized in the region of the Poynting vector energy minimum value.

The particles continue to move there with the velocity, which is determined by the force whose components are the scattering and absorbing components of the optical force and the viscous friction force. The results of simulation fully justify the results of the experiment suggested in [19]. At the same time the information about the gradient force value, which is determined by the gradient volume density and the degree of coherence of superposing fields, is lost in the general picture of particle motion.

According to our mathematical model (exp. 5), to make a quantitative estimation of the degree of coherence of the optical field, we choose such particles, in which the gradient component of the optical force will be the determining component of the motion. Let us analyze the behavior of the particles about $1 \mathrm{~nm}$ in size. These are the particles that can be defined as Rayleigh particles. As the simulation showed, when taking into account all the components of the resulting optical force, the gradient force value gets much greater than the values of other components. That is why the field gradient is able to move the particles into the maximum region, where the 


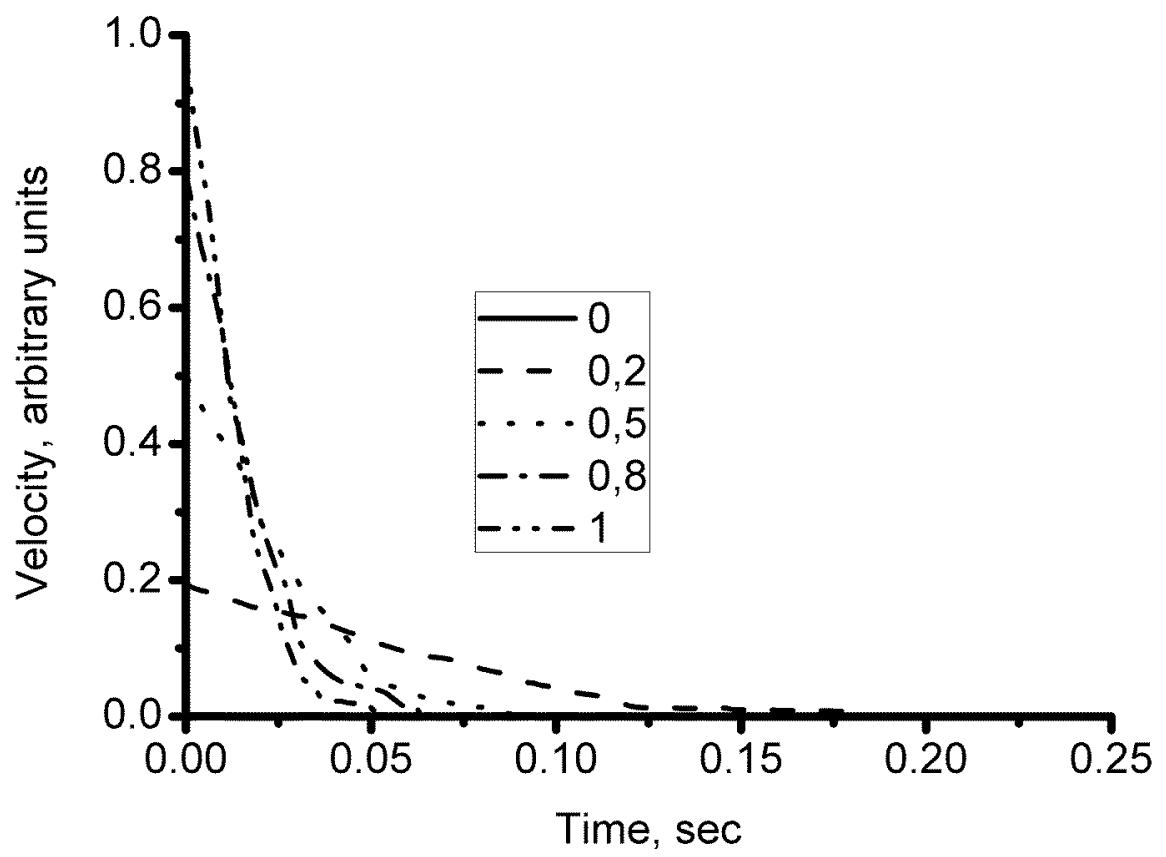

Fig. (4). The change of the normalized value of the averaged motion velocity of Rayleigh particles (the particles above $\lambda / 100)$ with time and with the change of the degree of coherence of interacting waves $\left(\eta^{(1,2)}\right)$ : the legend shows degrees of coherence that correspond to different curves.

trapping takes place. When the homogeneous optical field results from incoherent interaction of two superposing fields (the distribution gradient of the energy value is absent) the optical field does not influence the particles (Fig. 3).

A greater modulation depth of the energy volume density corresponds to a greater value of the degree of coherence, and, consequently, the degree of the energy inhomogeneity of the optical field increases. Correspondingly, the influence of the gradient component of the optical force increases (Fig. 3).

The value of the transverse component of the optical force is sufficient to trap the nanoparticles and keep them in the "capture" zone. In this case, according to the initial position of the corresponding nanoparticles, the velocity with which the particles are trapped by the field will be different. The velocity value is also influenced by the gradient value of the energy volume density.

Let us compare the results of calculation of the optical force acting on the particles of two different sizes at interaction of light with which various mechanisms of light scattering are realized. The calculation results are presented in Figs. (2 and 3). The principal difference between the results is observed in different course of the curves when the degree of coherence is changed and is connected with different direction of the effect of resultant forces in two situations under consideration. The insufficient impact of the gradient force (Fig. 2) in the case of the particles $100 \mathrm{~nm}$ in size on the value of the resultant optical force sets the distribution that is practically not related to the gradient of the volume energy density and, thus, to the degree of waves coherence. Naturally, for performing the following simulation experiments the particles $\lambda / 100$ in size were chosen, for which the effect of the gradient optical force was prevailing. The method of diagnosing the coherent properties of the interacting fields (waves) appeared to be especially effective for this particles ensemble.

The motion velocity of nanoparticles depends on the distribution of the energy volume density in the observation plane, and, accordingly, on the degree of coherence of superposing waves. The change of the degree of coherence causes the change of the normalized value of the averaged velocity of particle redistribution and their trapping in the optical field under the affect of optical forces (Fig. 4). The velocity graph presented in Fig. (4) is derived from Eq. (5). It describes the normalized value of the averaged particle motion velocity with time.

The maximum value of this velocity and, respectively, the maximum value of the resulting force are unequivocally related to the degree of coherence of the initial superposing beams, which form the optical field under investigation. For instance, the maximum normalized value of the averaged particle motion velocity is 0.8 (Fig. 4), the degree of coherence value is 0.8 . Any changes of the degree of coherence of the superposing waves cause the change of both the normalized value of the averaged motion velocity and the maximum value of the velocity of particle "trapping" into the maximum energy region in the analyzed field. Thus, the maximum normalized value of the averaged particle "trapping" velocity 0.5 corresponds to the degree of coherence 0.5 .

As it is shown by the results of the computer simulation, the particles are practically immediately "trapped" into the region of the maximum gradient value of the Poynting vector. As a result, the maximum normalized value of the averaged motion velocity is realized at the initial moments of time. At the same time, nanoparticles, possessing a certain mass, are characterized by some, say, insignificant inertia. Due to this, after "trapping" a certain period of time is needed, a period of "relaxation", for the particles to take a 


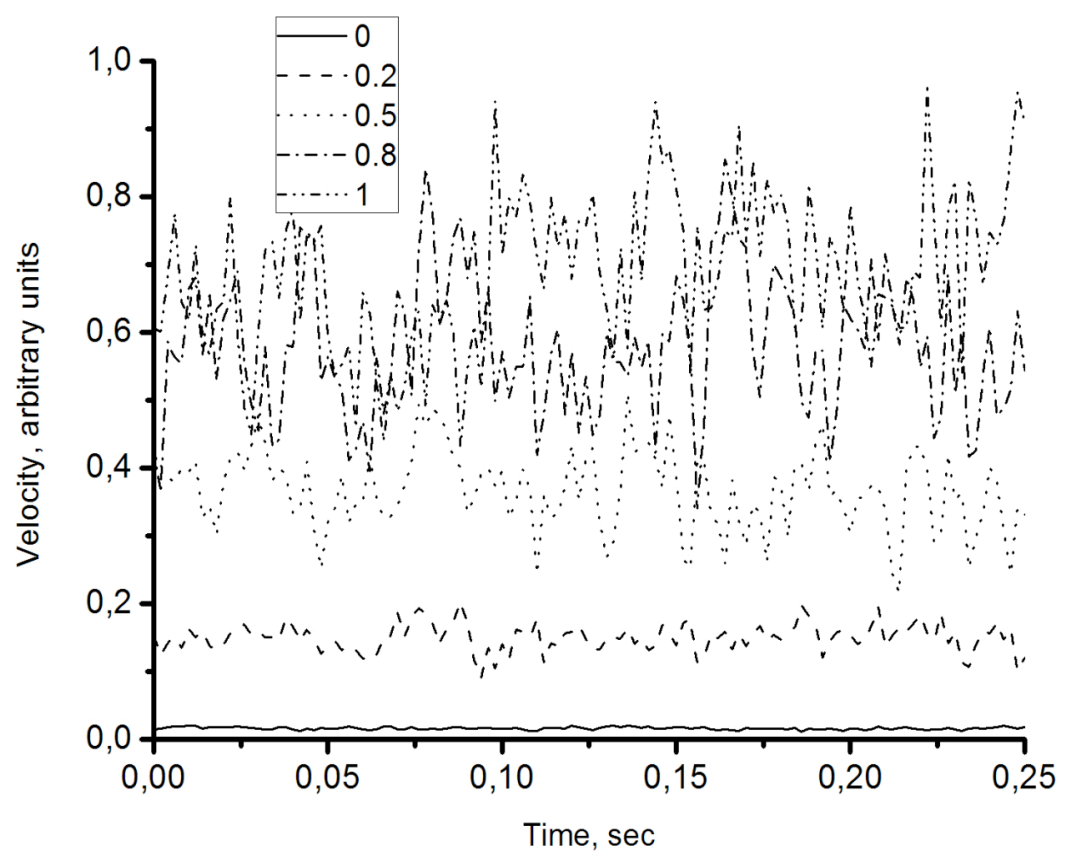

Fig. (5). The change of the normalized value of the averaged motion velocity of Rayleigh particles (the particles above $\lambda / 100$ ) with time and with the change of the degree of coherence of interacting waves $\left(\eta^{(1,2)}\right)$, when the Brownian motion of particles is taken into consideration: the legend shows degrees of coherence that correspond to different curves.

stable position. It is explained, firstly, by the progressive decrease of the velocity after "trapping", and, secondly, by the fact that the maximum motion velocity is chosen as the estimating criterion of the gradient of the field imhomogeneity and, consequently, of the degree of coherence.

The optical force, which causes the trapping of particles by the field and the normalized value of the averaged velocity particle motion are related directly to the degree of coherence of interacting fields. In this case the particle motion velocity is rather easily measured and can be chosen as an estimating parameter of the degree of coherence of interacting fields.

At the same time, when random fluctuations of particle motion caused by Brownian force action is taken into consideration, the uniqueness of the particle motion velocity and the degree of coherence of the optical field is lost (Fig. 5). The calculation of the resultant force acting on the investigated particles with consideration of the effect of Brownian force was performed according to the model presented in [37]. Brownian motion disguises the information about the coherence and polarization properties preset in the optical field under investigation.

\section{CONCLUSIONS}

Possibility of using the tested particles as additional instruments for estimating the degree of coherence of mutually orthogonal linearly polarized plane waves is demonstrated in accordance with the theoretical model of calculating the motion dynamics of the above-mentioned particles. Particles about $\lambda / 100$ in size are chosen to confirm the direct relation between the motion velocity and the degree of coherence of the mutually orthogonal waves, linearly-polarized in the incidence plane. The way of estimating the degree of coherence suggested in this paper is one more step forward in the theory of coherence. The discussed remote-sensing of an energy inhomogeneous optical field allows not only to estimate the coherence properties of optical fields, but opens up new possibilities for nanoparticle manipulation, creating optical tweezers and micromachines.

\section{CONFLICT OF INTEREST}

The authors confirm that this article content has no conflicts of interest.

\section{ACKNOWLEDGEMENTS}

Declared none.

\section{REFERENCES}

[1] Demergis V, Florin E-L. Ultrastrong Optical Binding of Metallic Nanoparticles. Nano Lett 2012; 12(11): 5756-60.

[2] Wang L-G, Zhao C-L, Wang L-Q, Lu X-H, Zhu S-Y. Effect of Coherence on Radiation Forces acting on a Rayleigh Dielectric Sphere. Opt Lett 2007; 32(11): 1393-5.

[3] Angel'skiı OV, Ushenko AG, Arkhelyuk AD, Ermolenko SB, Burkovets DN, Ushenko YuA. Laser polarimetry of pathological changes in biotissues. Proc SPIE 2000; 89(6): 1050-5.

[4] Angelsky OV, Ushenko AG, Ushenko YA, Ushenko YG. Polarization singularities of the object field of skin surface. J Phys Appl Phys 2006; 39(16): 3547.

[5] Angelsky OV, Demianovsky GV, Ushenko AG, Burkovets DN, Ushenko YA. Wavelet analysis of two-dimensional birefringence images of architectonics in biotissues for diagnosing pathological changes. J Biomed Opt 2004; 9, 4: 679-90.

[6] Angelsky OV, Tomka YuY, Ushenko AG, Ushenko YeG, Yermolenko SB. 2-D tomography of biotissue images in pre-clinic diagnostics of their pre-cancer states. Proc SPIE 2005; 5972: 59720N

[7] Angel'skiǐ OV, Ushenko AG, Ermolenko SB, et al. Polarizationbased visualization of multifractal structures for the diagnostics of pathological changes in biological tissues. Opt Spectrosc 2000; 9(5): 799-804

[8] Angel'skiĩ OV, Ushenko AG, Arkhelyuk AD, Ermolenko SB, Burkovets DN. Scattering of laser radiation by multifractal biological structures. Opt Spektrosc 2000; 88(3): 495-8. 
[9] Angelsky OV, Tomka YuY, Ushenko AG, Ushenko YeG, Yermolenko SB, Ushenko YuA. 2-D tomography of biotissue images in pre-clinic diagnostics of their pre-cancer states. SPIE Proc 2005; 5972: 158-62

[10] Ashkin A. Acceleration and trapping of particles by radiation pressure. Phys Rev Lett 1970; 24 : 156-9.

[11] Ashkin A, Dziedzic JM, Bjorkholm JE, Chu S. Observation of a single-beam gradient force trap for dielectric particles. Opt Lett 1986; 11: 288-90.

[12] Chen CH, Tai PT, Hsieh WF. Bottle Beam from a Bare Laser for Single-Beam Trapping. Appl Opt 2004; 43(32): 6001-6.

[13] Garcés-Chávez V, Roskey D, Summers MD, et al. Optical levitation in a Bessel light beam. Appl Phys Lett 2004; 85: 4001-3.

[14] Ye JY, Chang G, Norris TB, et al. Trapping cavitation bubbles with a self-focused laser beam. Opt Lett 2004; 29: 2136-8

[15] Okamoto K, Kawata S. Radiation Force Exerted on Subwavelength Particles near a Nanoaperture. Phys Rev Lett 1999; 83: 4534 -7.

[16] Volpe C, Quidant R, Badenes G, Petrov D. Surface plasmon radiation forces. Phys Rev Lett 2006; 96: 238101.

[17] Angelsky O, Mokhun A, Mokhun I, Soskin M. The relationship between topological characteristics of component vortices and polarization singularities. Opt Commun 2002; 207(1): 57-65.

[18] Angelsky OV, Burkovets DN, Maksimyak PP, Hanson SG. Applicability of the singular-optics concept for diagnostics of random and fractal rough surfaces. Appl Opt 2003; 42(22): 4529-40.

[19] Angelsky OV, Gorsky MP, Maksimyak PP, Maksimyak AP, Hanson SG, Zenkova CYu. Investigation of optical currents in coherent and partially coherent vector fields. Opt Express 2011; 19(2): 66072.

[20] Angelsky OV, Polyanskii PV, Mokhun II, et al. Optical Measurements: Polarization and Coherence of Light Fields. Modern Metrology Concerns. NY: InTech 2012.

[21] Angelsky OV, Zenkova CYu, Gorsky MP, Gorodyns'ka NV. Feasibility of estimating the degree of coherence of waves at the near field. Appl Opt 2009; 48(15): 2784-8.

[22] Angelsky OV, Hanson SG, Zenkova CYu, Gorsky MP, Gorodyns'ka NV. On polarization metrology (estimation) of the degree of coherence of optical waves. Opt Express 2009; 17(18): 1562334.

[23] Ashkin A. Optical Trapping and Manipulation of Neutral Particles Using Lasers. Singapore: Hackensack; NJ: World Scientific 2006.

[24] Bekshaev AYA, Angelsky OV, Sviridova SV, Zenkova CYU. Mechanical Action of Inhomogeneously Polarized Optical Fields and Detection of the Internal Energy Flows. Adv Opt Technol 2011;2011:11
[25] Angelsky OV, Bekshaev AYA, Maksimyak PP, Maksimyak AP, Hanson SG, Zenkova CYU. Self-diffraction of continuous laser radiation in a disperse medium with absorbing particles. Opt Express 2013; 21(7): 8922-38.

[26] Dienerowitz M, Mazilu M, Reece PJ, Krauss TF, Dholakia K. Optical vortex trap for resonant confinement of metal nanoparticles. Opt Express 2008; 16(7): 4991-48.

[27] Angelsky OV, Dominikov NN, Maksimyak PP, Tudor T. Experimental revealing of polarization waves. Appl Opt 1999; 38(14): 3112-7.

[28] Angelsky OV, Bekshaev AYA, Maksimyak PP, Maksimyak AP, Hanson SG, Zenkova CYU. Orbital rotation without orbital angular momentum: mechanical action of the spin part of the internal energy flow in light beams, Optics Express 2012; 20(4): 3563-71.

[29] Angelsky OV, Bekshaev AYA, Maksimyak PP, et al. Circular motion of particles suspended in a Gaussian beam with circular polarization validates the spin part of the internal energy flow. Opt Express 2012; 20(10): 11351-6.

[30] Zenkova CYU, Gorsky MP, Soltys IV, Angelsky PO. On the possibilities of using inhomogeneity in light energy distribution for estimating the degree of coherence of superposing waves. Appl Opt 2012; 51(10): C38-43.

[31] Angelsky OV, Yermolenko SB, Zenkova CYU, Angelskaya AO. Polarization manifestations of correlation (intrinsic coherence) of optical fields. Appl Opt 2008; 47(29): 5492-9.

[32] Ellis J, Dogariu A, Ponomarenko S, Wolf E. Degree of polarization of statistically stationary electromagnetic fields. Opt Commun 2005; 248: 333-7.

[33] Mujait M, Dogariu A, Wolf E. A law of interference of electromagnetic beams of any state of coherence and polarization and the Fresnel-Arago interference laws. J Opt Soc Am 2004; 21(12): 2414-7.

[34] Zenkova CYU, Gorsky MP, Maksimyak PP, Maksimyak AP. Optical currents in vector field. Appl Opt 2011; 50(8): 1105-12.

[35] Zenkova CYU, Gorsky MP, Soltys IV, Angelsky PO. Use of motion peculiarities of test particles for estimating degree of coherence of optical fields. Ukr J Phys Opt 2012; 13: 183-95.

[36] Palik ED. Handbook of Optical Constants of Solids. San Diego: Academic Press 1985.

[37] Zenkova CYU, Gorsky MP, Soltys IV, Angelsky PO. Influence of the Brownian movement on the motion of nano and micro-particles in the inhomogeneous optical field. Opt Electron Rev 2012; 20, 3: 247-54.

Received: March 21, 2013

Revised: May 13, 2013

Accepted: May 13, 2013

(C) Angelsky et al.; Licensee Bentham Open.

This is an open access article licensed under the terms of the Creative Commons Attribution Non-Commercial License (http://creativecommons.org/licenses/by-nc/3.0/) which permits unrestricted, non-commercial use, distribution and reproduction in any medium, provided the work is properly cited. 